\title{
Monitoring of slowly progressing deterioration of computer numerical control machine axes
}

\author{
E Uhlmann $^{1,2}$, C Geisert ${ }^{1 *}$, and E Hohwieler ${ }^{2}$ \\ ${ }^{1}$ Fraunhofer Institute for Production Systems and Design Technology (IPK), Berlin, Germany \\ ${ }^{2}$ Department of Manufacturing Technology, Technische Universität Berlin, Berlin, Germany
}

The manuscript was received on 1 November 2007 and was accepted after revision for publication on 18 March 2008.

DOI: $10.1243 / 09544054 J E M 1040$

\begin{abstract}
The feed axes of computer numerical control (CNC) grinding machine tools are among the most mechanically stressed components of machine tools owing to the high process forces and rough manufacturing environment which they encounter. The resulting wear and tear depends strongly on the product range and the manner of machine operation. To counteract a functional deficiency of these central machine units, the current usual approach is preventive maintenance. The manual inspection of feed axes is complex and time consuming. A complicating matter is that the deterioration normally progresses very slowly and depends on the position of the stress along the axis. Existing approaches to automated estimation of the 'health status' of feed axes do not take this factor into account.

This paper presents a procedure that addresses this gap. During simple test routines, the drive current, axis position, and feed rate are recorded. With the help of additional machine data, characteristic values are computed directly at the computer of the human-machine interface (HMI). The results are then transferred to and stored on a database server at the machine manufacturer. This approach enables the service technician to trace the progression of the axes' 'health status' over a long time. This approach makes it possible to detect trends in the characteristic values at an early point in time. This leads to a better planning of necessary maintenance actions adapted to the remaining lifetime of the wearing component.
\end{abstract}

Keywords: condition monitoring, availability, maintenance, wear and tear, feed axes, life-cycle

\section{INTRODUCTION}

Applications of centreless external cylindrical grinding are exceedingly appropriate to mass production. One of the main components of such computer numerical control (CNC) machine tools to experience wear and tear is the feed axes. How fast and where along the axis deterioration occurs depends on factors such as:

(a) the tool-workpiece combination;

(b) the grinding method;

(c) the condition of lubrication;

(d) the quality of maintenance procedures.

Grinding methods such as plunge-cut grinding and throughfeed grinding (see Fig. 1) in particular involve a very limited effective workspace.

*Corresponding author: Department of Manufacturing Technology, Pascalstrasse 8-9, Berlin, Germany. email: claudio. geisert-projekt@ipk.fraunhofer.de
This leads to stress on the feed axis, which is highly location-specific. At these locations along the axis, wearout can be expected to occur more rapidly and to a greater extent. Another factor that influences the progress of deterioration is the contamination of the guidance by grinding debris. This happens if the wiper does not function properly. The result is a loss of accuracy or, in the worst case, a breakdown of the feed axis [1]. As explained in reference [2], non-working wipers are responsible for 90 per cent of all failures in linear guidance.

Owing to the influence of the axis controller, the deterioration of a feed axis only becomes obvious when its function fails and an alarm message is generated by the programmable logic controller (PLC). In this article, a procedure for tracing changes in the dynamic behaviour of feed axes is described. Using statistical signal processing methods, characteristic values are computed. These can be used to determine the axis 'health status'. A history of 


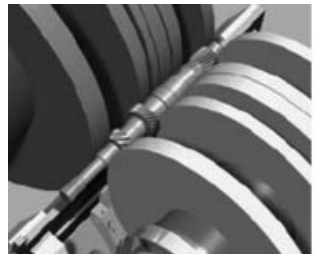

(a)

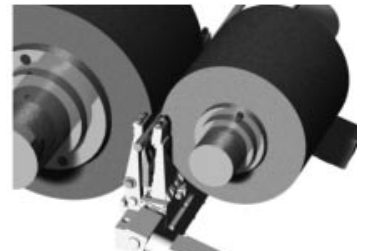

(b)
Fig. 1 (a) Plunge-cut grinding and (b) throughfeed grinding (copyright Studer Mikrosa GmbH)

position-dependent characteristic values is generated and stored in a relational database.

\section{STATE OF THE ART}

Much effort concerning condition monitoring systems focuses on the research and development of IT-frameworks with the ability to integrate external sensor signals respectively to use control-integrated signals [3-8]. However, even though most of these systems provide various methods for signal processing and data analysis, it is difficult to adapt them to the system that needs to be monitored [9]. Given a particular recorded pattern, expert know-how and the results of multiple case studies are necessary to ensure that the deterioration status is interpreted correctly.

This section considers a selection of commercial products and findings in current research on feed axis monitoring.

\section{1 ePS Network Services}

With its ePS Network Services, the Siemens Corporation offers a tool for the acquisition and documentation of the current machine axis state [10]. This feature is called eP-Performance and includes the following tests:

(a) the circularity test;

(b) the synchronized axes test;

(c) the universal axis test.

During the tests, control internal data are sampled and used to calculate certain parameters. The parameters calculated in the universal axis test are characteristic quantities to define the friction, the moment of inertia and a torque offset [11]. These are obtained using complex mathematical models [12] in which the dynamic system is stimulated by a special axis motion profile.

Repetitions of single measurements are used to generate trend curves from characteristics in measurement series. It is suggested to use these trend curves as a basis for the optimization of maintenance activities (see Fig. 2).

There are two additional features implemented, called NC monitor and PLC monitor. The NC monitor

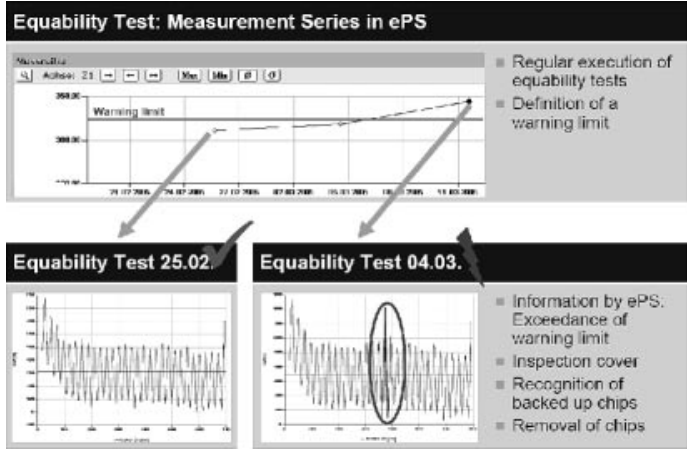

Fig. 2 Trend generation and monitoring with ePS Network Services [10] (copyright Siemens AG 2006)

can be used to estimate the life-cycle loads of machine axes by counters, while the PLC monitor enables long time records of arbitrary PLC variables [11].

\subsection{LoeWe - life cycle oriented machine tool}

The project LoeWe [13] was funded by the German Federal Ministry of Education and Research (BMBF) within the framework of the 'Research for Tomorrow's Production' programme (project duration 2004-2007). One objective of this research project was to monitor the deterioration status of components of particular functional relevance. The ball screw drive was identified as mainly responsible for the axis positioning accuracy. Increasing wear of the ball screw leads to a decrease of the ball screw pre-load. Therefore a condition monitoring strategy for the detection of loss of this pre-load was chosen [14]. The implemented methods for measuring the wear of the ball screw are based on investigations that are described in reference [15]. In the first method the change of the natural frequency of the axis in dependency to pre-load conditions is analysed. Figure 3 shows that the estimated eigenfrequency falls with a decrease of the ball screw pre-load.

The second method uses the spring characteristics of ball screws that differ for unworn and worn ball

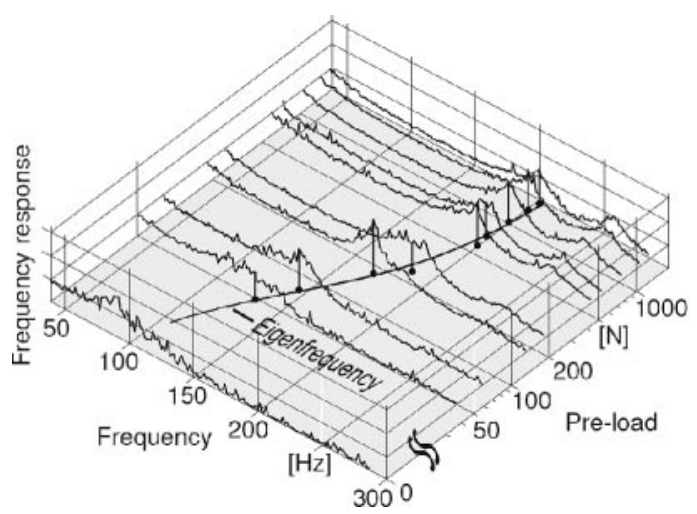

Fig. 3 Correlation between pre-load and eigenfrequency [14] (copyright IFW) 
screws. Further wear measurements for vertical feed axes are planned using a specific velocity profile [14].

\subsection{REACH - development of a method to improve the reliability and availability of machine tools}

A modular system for the monitoring of machine tool component state was developed by the European Brite/EuRam project REACH (project duration 1998-2001) [16]. Current control internal signals of open CNCs were used to describe the behaviour of the components. If necessary, external sensors were integrated into the system [17].

With the aid of long-running tests, it was shown that the use of the drive current instead of the displacement force signal is sufficient for detecting mechanical disturbances. Validation of the realized monitoring system was done at a test bench for two typical types of feed axis failure:

(a) backlash;

(b) pitting on guideways.

Backlash detection was analysed by building the difference between the directly measured position and the position calculated from the motor encoder. For the detection of pitting the drive current signal was chosen [18].

In the context of this project, a doctoral thesis warrants mentioning [19]. A detailed enumeration of the analytical methods used to detect various feed axis disturbances is provided in this work.

\subsection{Machine and process diagnostics}

At the Institute for Control Engineering of Machine Tools and Manufacturing Units (ISW) in Stuttgart, the usability of control-integrated signals for the condition monitoring of feed axes is currently undergoing research. The investigations, which where carried out in this context were funded by the Research Association for Machine Tools and Manufacturing Technologies (FWF) and the German Research Foundation (DFG).

With the help of adapted test signals the 'Stribeck curve' can be estimated [20]. Furthermore, methods for the condition monitoring of ball screws, commonly used components within feed axes, were developed. Algorithms for the estimation of characteristic values were implemented at the ISW [21] for:
(a) change of lead screw pitch error;
(b) friction;
(c) stiffness;
(d) backlash error.

\section{5 e-Industrial Services}

One aspect of the Fraunhofer Institute's research project 'e-Industrial Services' (2000-2003) was the

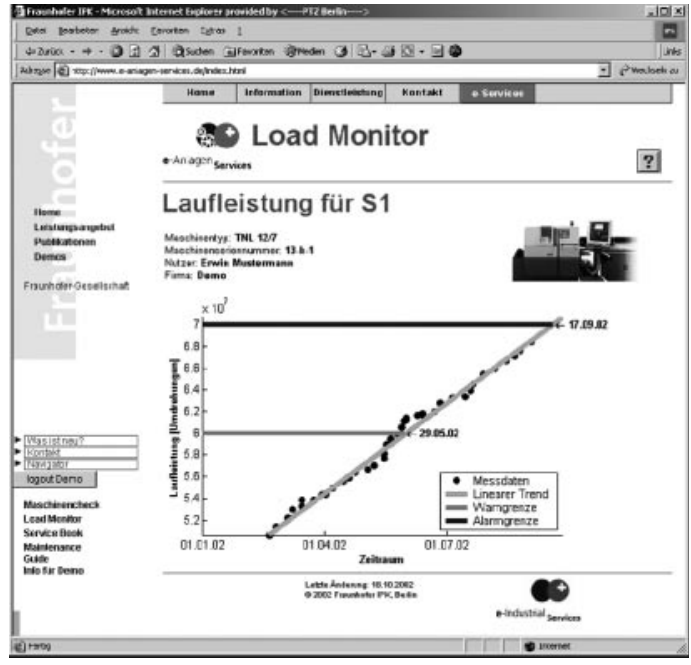

Fig. 4 Presentation of life cycle data (here: cumulated rotation speed) as a Web Service (copyright Fraunhofer IPK)

development of electronic services for the analysis and prediction of machine health status using enhanced diagnostic algorithms [22].

In this approach, data are recorded during a specifically designed test-NC-procedure under specifically defined conditions. The signals recorded and used are the drive current and the rotation speed of the axis or the spindle drives. A linear mathematical model of this electromechanical unit was used to estimate the condition of a feed axis with controlled rotation speed. From this system of differential equations characteristic diagnostic features were generated. These features represent the static and sliding friction parameters and the moment of inertia. The drive current, rotation speed and acceleration derived from the rotation speed are used as input for the least squares method. This approach is used for parameter identification in the case of an over-determined set of linear equations [23].

In addition to the diagnostic tests, the load profile of the machine tool is continuously logged during normal machine operation. From the load profile experience-based statements about the expected 'health state' can be made. Figure 4 shows an example of an internet based service that indicates the point in time at which service activities are expected to be necessary $[\mathbf{2 4}]$.

\section{MONITORING CONCEPT}

As mentioned in section 1, in the case considered, the machine tool axes underlay a special load profile that is specific to this kind of machining. The challenge is to develop a monitoring system which exhibits on the one hand sufficient sensitivity in order to detect 
progressive, position-dependent deterioration, and on the other hand sufficient robustness to endure the rough manufacturing environment. Last but not least, the monitoring system must fulfil the requirement that additional auxiliary process time be avoided.

\subsection{Premises}

Centreless grinding machines are mainly used for mass production in the automotive industry. The machine tools are characterized by a great number of feed axes owing to the centreless grinding technology. A typical axes plan is shown in Fig. 5. The grinding wheel made of corundum is mounted on the X1-axis. The weight of a new, unused wheel is about $400 \mathrm{~kg}$ (without mounting device) and slowly decreases as a result of abrasion.

The machine tools are equipped with an open CNC system of type SINUMERIK 840D made by Siemens. The open CNC architecture enables data acquisition of control internal sensor signals and machine data via OPC (OLE for Process Control).

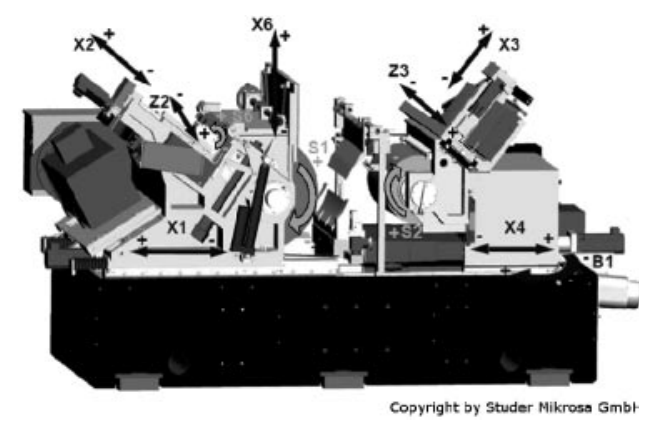

Fig. 5 Axes plan of a MIKROSA centreless grinding machine tool of type KRONOS L (copyright Studer Mikrosa $\mathrm{GmbH}$ )

\subsection{Structure of the monitoring system}

The proposed monitoring system is capable of the following principal tasks:
(a) automated execution of axis tests;
(b) data logging;
(c) signal pre-processing (sensor signals only);
(d) data compression;
(e) data transmission;
(f) data storage;
(g) data analysis;
(h) report generation.

Figure 6 shows the system's scheme. A decentralized architecture was chosen with the multiplicity of the tasks in mind. While data logging, signal preprocessing, and data compression take place at the machine tool, storage, analysis and visualization take place at the location of the machine manufacturer. This separation of location has the advantage that a large amount of data, even coming from separate machine tools, can easily be used for comparative analyses. Long-term trend analyses of archived axis test results support the detection of hidden trends in the dynamic behaviour of feed axes. Trend analysis is an important tool owing to the controller's influence on the dynamics of feed axis. The axis function will be correct as long as control variables are regulated within their limitations. Trend monitoring of characteristic values, which are generated from implemented axis tests can be used to uncover small trends, caused by for example deterioration of feed axes, even before the effects become obvious.

The aspect of data transmission will not be addressed in this paper. It belongs to the area of information and communication technologies and secure IT infrastructure build-up. A comprehensive discussion of these subjects can be found in reference [25].

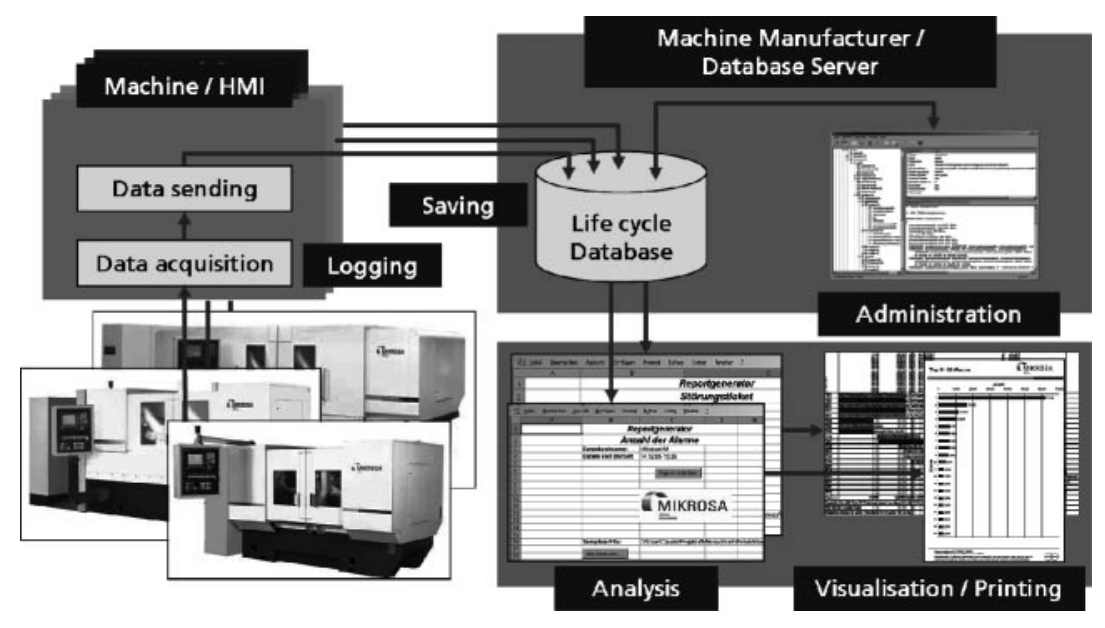

Fig. 6 Global structure of the monitoring system 


\subsection{Axis test and life cycle data}

If wear and tear of a feed axis increases, the dynamic behaviour of the feed drive system changes. For example, as a result of modifications to the tribological system, friction rises and sluggishness occurs. With the increased friction the drive current also rises [26].

By moving the axis with constant feed rate across its entire range (in both directions), it is possible to detect changes in the dynamical behaviour. During the test, the drive current, position and feed are sampled. Figure 7 shows the sampled position and drive current data of an axis test. The feed signal is used automatically to detect the two intervals in which the positive or negative axis motion is constant. The phases of acceleration are masked because they would interfere with the analysis.

Auxiliary data providing information on the life cycle of the machine tool are logged continuously. This includes the alarm-log history and information on previous maintenance activities. Such data facilitate failure analysis in detailing the events leading to a failure during operation [27].

\subsection{Pre-processing}

The following statistical moments are calculated from the drive current signal and used to generate key indicators for condition monitoring:

(a) mean: $\mu=\frac{1}{N} \sum_{i=1}^{N} X_{i}$

(b) variance: $\operatorname{Var}(X)=E\left((X-\mu)^{2}\right)$

(c) skewness: $v(X)=\frac{E\left((X-E(X))^{3}\right)}{\operatorname{Var}(X)^{3 / 2}}$

(d) excess kurtosis: $\gamma_{2}=\frac{E\left((X-E(X))^{4}\right)}{\operatorname{Var}(X)^{2}}-3$

The meaning of significant changes in the mean and variance is evident. The smoothness of the system is characterized by the variance of the signal, while the drive current is proportional to the driving torque. Therefore, difficulties experienced by the feed axis are reflected in respective trends in the mean.

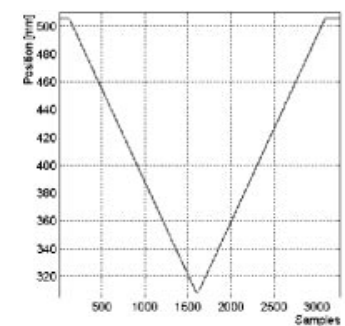

(a)

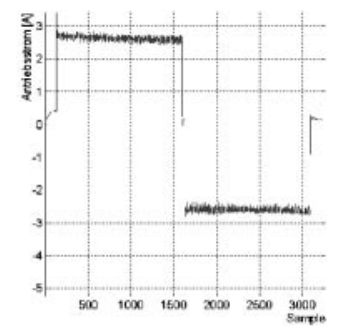

(b)
Fig. 7 (a) Axis position and (b) drive current

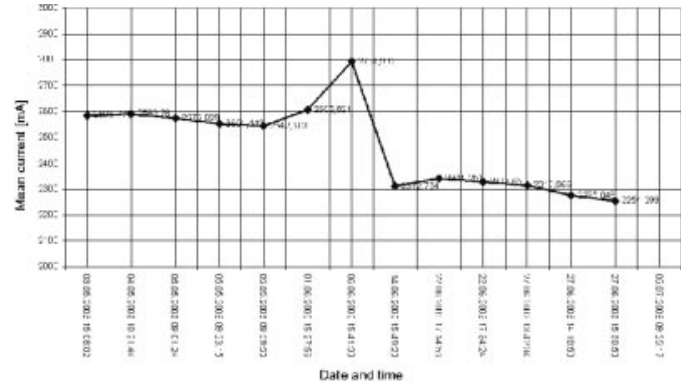

Fig. 8 Trend of feed drive current signal (copyright Fraunhofer IPK)

An exemplary trend of the mean current of the X1axes of a grinding machine of type KRONOS L is presented in Fig. 8. The data represent the dynamic state of the axes within a small area (position: 383-403 mm).

The general trend in the characteristic value is easy to see. Less power is needed to traverse the feed axis. The machine is still working, and no maintenance activities have been necessary up until now. A possible reason for this phenomenon is the shake down effect of new feed axes. After a certain period of usage the axes motion becomes smoother. An explication for peak in the centre of the trend cannot be given, owing to the fact that this machine is not located at the test field of MIKROSA.

The skewness indicates on which side of the mean the weight of the data falls. It can be used to detect onsetting failures that depend on the direction of traversing. The excess kurtosis is an indicator for variations of the distribution referring to normal distribution. Distributed local damages along the axes lead to a distribution with many peaks, for example pitting on guideways. In this case, the excess kurtosis is greater than zero [28].

As mentioned before, the deterioration does not proceed homogeneously at all points along the axis. For this reason, the analysis of the signal is divided into small 'windows'. Every window pertains to a certain position along the axis.

To avoid additional auxiliary process time, the axis test is combined with a periodically recurring traversing of the axes in which the guidance is provided with lubricant.

\subsection{Data management}

In order to detect trends in the recorded characteristic values, an 'initial state' must first be defined. The characteristic values in the initial state are in accordance with a 'good' feed axis state of health and provide the basis for trend detection.

XML (eXtensible Markup Language) was chosen as the data interface between the different components of the monitoring system. XML is a universal data exchange format that can be used for machine- 


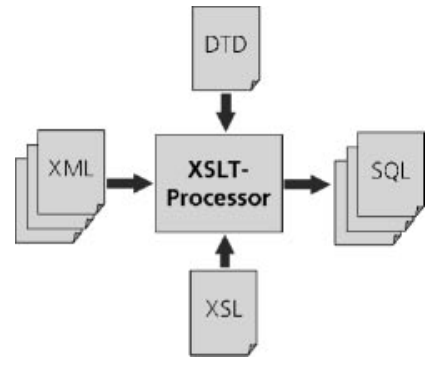

Fig. 9 Data transformation from XML to SQL

to-machine communication. In the work presented the XML-logfiles are parsed by an XSLT-processor. The processor generates files in SQL-format (Structured Query Language) as it imports the data into a relational database (see Fig. 9).

The scheme of the database shows the relations between the data, the components, and the machine tool. Visualization of the data is provided by predefined reports. It is possible to present visually the stored information for various time intervals in various ways.

\section{CONCLUSION AND OUTLOOK}

Using the proposed method of monitoring the condition of feed axes, it is possible to detect trends and slowly progressing changes in the dynamic system. Information on the machine tool's load history that is additionally logged during the machine tool's life cycle can be used to support the search for causes of failure. The database-based approach facilitates comparative analyses and long-term investigations. It is still an open task to provide evidence, which kind of axis deterioration and failures are detectable by observing the proposed characteristic values. At the moment, this is done by collecting experience in the field from operating machines.

Ongoing work at the Fraunhofer IPK deals with the development of model based machine diagnosis. Several mathematical models were implemented. The evaluation of the models is done using different motion profiles for the axis movement to stimulate the system's dynamic behaviour. The aim of this investigation is to generate further robust and sensitive characteristic values for the monitoring of feed axes.

With the implementation of intelligent machine tool components that are able to analyse and store their wear status the field of condition monitoring applications will increase.

\section{ACKNOWLEDGEMENTS}

Parts of the presented work have been carried out within the research project DYNAPRO funded by the
'Stiftung Deutsche Industrieforschung'. It was done in cooperation with Studer Mikrosa GmbH, a manufacturer of centreless external cylindrical grinding machine tools.

The Fraunhofer Institute for Production Systems and Design Technology is a partner of the EU-funded FP6 Innovative Production Machines and Systems (I*PROMS) Network of Excellence.

\section{REFERENCES}

1 Available online at www.ima.unistuttgart.de/dich tungstechnik/abgeschlossene_projekte/wzm/wzm.en. html. Institute of Machine Components, University of Stuttgart, 2002.

2 Jansen, M. Abstreifer für Werkzeugmaschinenführungen. Dissertation, Universität Stuttgart, URN: urn:nbn:de:bsz: 93-opus-24403, available online at URL: http://elib.uni stuttgart.de/opus/volltexte/2005/2440/, 2005.

3 Lee, J., Abujamra, R., Jardine, A. K. S., Lin, D., and Banjevec, D. An integrated platform for diagnostics, prognostics and maintenance optimization. Proceedings of the Intelligent Maintenance Systems 2004, 15-27 July 2004, Arles, France.

4 Lee, J. and Ni, J. Smart prediction to prevent downtime. Innovation, 2004, 4(3), 41-42.

5 Djurdjanovic, D., Lee, J., and Ni, J. Watchdog Agent an infotronics-based prognostics approach for product performance degradation assessment and prediction. Advd Engng Inf., 2003, 17(3-4), 109-125.

6 Grudzien, W. and Seliger, G. Life cycle unit in product life cycle - tool for improved maintenance, repair and recycling In Proceedings of 33rd CIRP International Seminar on Manufacturing Systems, Stockholm 5-7 June 2000, pp. 143-148 (International Institution for Production Engineering Research).

7 Hirschmann, J. Fault detection and diagnosis of the electromechanical drive units in the automation technology. Conference Proceedings: E-Manufacturing and E-Business Integration, Milwaukee, Wisconsin, USA, 9-11 September, 2002, pp. 179-181.

8 Middendorf, A., Buchholz, A., Hefer, J., Reichl, H., Schrank, K., and Seliger, G. Life-cycle information units for monitoring and identification of product use conditions. Proceedings of Global Conference on Sustainable Product Development and Life Cycle Engineering 2004, 29 September-1 October, 2004, Berlin, pp. 91-96.

9 Walter, K.-D. Wireless für Präventiv-Aufgaben. Computer \& Automation, 2006, 01, 36-39.

10 Heinz, J. ePS Network Services - internet based condition monitoring of CNC machines. Lecture held at the 'International Machine Tool Forum' at IMTEX 2007, Bangalore, India, 20 January 2007, Copyright (c Siemens AG 2006.

11 ePS Network Services, Function Manual (FH)-Release 04/2007, Copyright (c) Siemens AG 2007.

12 Barth, R. Produktiver mit Condition Monitoring. In: WB Werkstatt + Betrieb 09/2005, pp. 183-185 (Carl Hanser Verlag, Munich).

13 Available online at www.projekt-loewe.com 
14 Denkena, B., Harms, A., Jacobsen, J., Möhring, H.-C., Lange, D., and Noske, H. Life-cycle oriented development of machine tools. In Proceedings of the 13th CIRP International Conference on Life Cycle Engineering, LCE 2006, 31 May-2 June 2006, Katholieke Universiteit Leuven, Belgium, pp. 693-698.

15 Imiela, J. Verfügbarkeitssicherung von Werkzeugmaschinenachsen mit Kugelgewindetrieb durch modellbasierte Verschleißüberwachung. Doctoral Thesis, PZH-Verlag, Hannover, Germany, 2006.

16 Available online at http://www.cordis.lu/data/PROJ_ BRITE/ACTIONeqDndSESSIONeq23541200595ndDOCeq 115ndTBLeqEN_PROJ.htm. (accessed 1 November 2007).

17 Available online at http://www.cordis.lu/data/RESU_ BRITE/ACTIONeqDndSESSIONeq23864200598ndDOCeq 152ndTBLeqEN_RESU.htm. (accessed 1 November 2007).

18 Plapper, V. and Weck, M. Sensorless machine tool condition monitoring based on open NCs. Proceedings of the 2001 IEEE International Conference on Robotics and Automation, Seoul, Korea 21-26 May 2001, pp. 3104-3108.

19 Plapper, V. Steuerungsintegrierte Überwachung von Werkzeugmaschinen. Doctoral Thesis, Aachen, RWTH, 2004.

20 Dietmair, A., Walther, M., and Pritschow, G. Antriebsbasierte Maschinendiagnose - Fortschritte bei der Nutzung von Antriebssignalen zur Maschinendiagnose. wt Werkstattstechnik online, 2005, 95(5), 351-356.

21 Walther, M. and Verl, A. Maschinendiagnose auf Basis von Antriebssignalen. In Zuverlässigkeit und Diagnose in der Produktion, Fortschritt-Berichte VDI, Vol. No. 663, VDI Verlag, Düsseldorf, Germany, 2007, pp. 39-55.
22 Uhlmann, E., Hohwieler, E., and Berger, R. Providing online services for machine operation and maintenance. In Proceedings of the International IMS Forum 2004, Vol. 2: Global challenges in manufacturing, Villa Erba, Cernobbio, Italy, 17-19 May 2004, pp. 1120-1127.

23 Hohwieler, E., Berger, R., and Geisert, C. Condition monitoring services for e-Maintenance. In Proceedings of the 7th IFAC Symposium on Cost Oriented Automation, 7-9 June 2004, Gatineau/Ottawa, Canada.

24 Hohwieler, E. and Geisert, C. Intelligent machines offer condition monitoring and maintenance prediction services. In Proceedings of the 4th CIRP International Seminar on Intelligent Computation in Manufacturing Engineering (CIRP ICME '04), (Ed. R. Teti), 30 June-2 July, 2004, Sorrento, Italy, pp. 599-604.

25 Berger, R. Sicherheitszentrierte Architektur für Internetbasierte Dienste im Maschinen und Anlagenbau. Doctoral Thesis, Technische Universität, Berlin, 2005.

26 Krüger, J. Methoden zur Verbesserung der Fehlererkennung an Antriebsstrecken. Doctoral Thesis, Technische Universität, Berlin, 1998.

27 Hohwieler, E. and Geisert, C. Holistic approach for condition monitoring and prediction of machine axis. In Proceedings of First I*Proms Virtual Conference on Intelligent production machines and systems, 4-15 July 2005 (Eds D. T. Pham, E. E. Eldukhri, and A. J. Soroka), Elsevier, 2005, pp. 67-72.

28 Geisert, C. and Uhlmann, E. Steuerungsbasierte Erfassung zustandsrelevanter Life-Cycle Daten an CNCWerkzeugmaschinen. In Zuverlässigkeit und Diagnose in der Produktion, Fortschritt-Berichte VDI, Vol. 2, No. 663, VDI Verlag, Düsseldorf, Germany, 2007, pp. 1-14. 